ISSN-L 2077-0014

DOI: https://doi.org/10.33326/26176068.2021.3.1155

Artículo original/Original article/Artigo original

\title{
Conductas de riesgo e infecciones de transmisión sexual en mujeres en edad fértil
}

\author{
Risk behaviors and sexually transmitted infections in women of childbearing age \\ Comportamentos de risco e infecções sexualmente transmissíveis em mulheres em idade fértil
}

\author{
Evelyn L. Malca Herrera ${ }^{1, a, b}$ \\ Juan José Changlio Roas ${ }^{2, c, d}$
}

\author{
(iD https://orcid.org/0000-0002-4933-0778 \\ (iD https://orcid.org/0000-0001-9063-3757
}

\begin{abstract}
Resumen
Objetivo: Evaluar las conductas de riesgo y las infecciones de transmisión sexual en mujeres en edad fértil en dos establecimientos del primer nivel de atención de Tacna, Perú. Material y métodos: Estudio no experimental, transversal, en 177 mujeres en edad fértil atendidas en los centros de salud La Esperanza y San Francisco, seleccionadas al azar, a quienes se aplicó un cuestionario para medir las infecciones de transmisión sexual desarrollado por el Ministerio de Salud (MINSA) del Perú, previo consentimiento informado. Se presentó tablas comparativas, para el análisis se utilizó el estadístico Chi cuadrado. Resultados: Son conductas de riesgo para contraer infecciones de transmisión sexual, el número de parejas sexuales, tener relaciones coitales con una pareja que ha tenido varias parejas sexuales $(p<0,05)$. Del total de mujeres en edad fértil que presentan síndrome de flujo vaginal (48,59\%), el 64,29\% pertenecen al Centro de Salud San Francisco y el 34,41 \% al Centro de Salud La Esperanza. Se halló una diferencia significativa entre las conductas de riesgo de las mujeres de ambos centros de salud. $(p<0,05)$. Conclusión: Las conductas de riesgo y las infecciones de transmisión sexual en mujeres en edad fértil en dos establecimientos del primer nivel de atención de Tacna son diferentes.
\end{abstract}

Palabras clave: conductas, riesgo, infecciones de transmisión sexual, mujeres en edad fértil

\begin{abstract}
Objective: To assess risk behaviours and sexually transmitted infections in women of childbearing age in two primary care facilities in Tacna, Peru. Material and methods: Non-experimental, cross-sectional study of 177 women of childbearing age attended at La Esperanza and San Francisco health centers, randomly selected to whom a questionnaire to measure sexually transmitted infections developed by the Ministry of Health (MINSA) of Peru was applied, with informed consent. Comparative tables are presented, the Chi-squared statistic was used for the analysis. Results: They are risk behaviors to get sexually transmitted infections, the number of sexual partners, have intercourse with a partner who has had several sexual partners $(p<0.05)$. Of the total number of women of childbearing age with vaginal discharge syndrome (48.59 \%), $64.29 \%$ belong to the San Francisco Health Center and $34.41 \%$ to La Esperanza Health Center. A significant difference was found between the risk behaviors of women in both health centers $(p<0.05)$. Conclusion: Risk behaviours and sexually transmitted infections in women of childbearing age in two primary care facilities in Tacna are different.
\end{abstract}

Keywords: behaviors, risk, sexually transmitted infections, women of childbearing age

\footnotetext{
${ }^{1}$ Red de Salud Tacna. Gerente del CLAS 5 y 6 La Yarada. Tacna, Perú

${ }^{2}$ Región de Salud de Tacna. Director Ejecutivo de Medicamentos. Tacna, Perú

a Obstetra

${ }^{\mathrm{b}}$ Magister en Salud Pública

c Químico Farmacéutico

d Doctor en Epidemiología
} 


\begin{abstract}
Resumo
Objetivo: Avaliar os comportamentos de risco e as infecções sexualmente transmissíveis em mulheres em idade fértil em dois estabelecimentos do primeiro nível de atendimento de Tacna, Peru. Material e métodos: Estudo não experimental, transversal, em 177 mulheres em idade fértil atendidas nos centros de saúde La Esperanza e San Francisco, selecionadas aleatoriamente a quem foi aplicado um questionário para medir as infecções sexualmente transmissíveis desenvolvido pelo Ministério da Saúde (MINSA) do Peru, com o consentimento informado. São apresentados quadros comparativos, para a análise foi utilizado o estatístico Qui-quadrado. Resultados: São comportamentos de risco para contrair infecções sexualmente transmissíveis, o número de parceiros sexuais, ter relações sexuais com um casal que teve vários parceiros sexuais $(p<0,05)$. Do total de mulheres em idade fértil que apresentam síndrome de corrimento vaginal (48,59 \%), 64,29 \% pertencem ao Centro de Saúde São Francisco e 34,41 \% ao Centro de Saúde La Esperanza. Foi encontrada uma diferença significativa entre os comportamentos de risco das mulheres dos dois centros de saúde $(p<0,05)$. Conclusão: Os comportamentos de risco e as infecções sexualmente transmissíveis em mulheres de idade fértil em dois estabelecimentos do primeiro nível de atenção de Tacna são diferentes.
\end{abstract}

Palavras-chave: comportamento, risco, infecções sexualmente transmissíveis, mulheres em idade fértil

\section{Introducción}

La diseminación de las infecciones de transmisión sexual (ITS) en todo el mundo se ha convertido en un reto para la salud pública. Las ITS presentan una prevalencia cada vez mayor y causan problemas de salud que afectan a los hombres, las mujeres y los niños, incluso los que están por nacer. Los efectos de algunas ITS incluyen cáncer cervical, demencia y muerte. ${ }^{1}$

En el Perú, cuatro de cada diez hombres y cinco de cada diez mujeres tienen una ITS sin saberlo. Se calcula que anualmente se diagnostican más de 300 millones de nuevos casos de ITS en el mundo, casi diez veces la población del Perú. ${ }^{2}$

La OMS asegura que cada día más de un millón de personas de 15 a 49 años contraen infecciones de transmisión sexual curable. En total, al año se registran 376 millones de nuevos casos de estas cuatro infecciones: clamidiosis, gonorrea, tricomoniasis y sífilis. ${ }^{3}$

En muchas partes del mundo, las ITS son causas principales de infertilidad, tanto en el hombre como en la mujer. Algunas de las ITS afectan al feto y al recién nacido; asimismo, los embarazos de mujeres que padecen estas enfermedades pueden terminar en una muerte fetal o neonatal o en nacimiento de un neonato con alguna morbilidad. Muchos estudiosos, sostiene que la diseminación de las ITS depende de factores como: el comportamiento y las actitudes sexuales, el incremento de las parejas sexuales, la disponibilidad de servicios para el diagnóstico temprano y la identificación de los contactos; y los cambios en la virulencia y sensibilidad de los organismos causantes de las ITS. ${ }^{1}$
Existen diversos estudios que evidencian esta problemática, como el realizado en Cuba, en el 2017, sobre conductas sexuales de riesgo asociadas a las infecciones de transmisión sexual en adolescentes de una comunidad, dando el siguiente resultado: el mayor número estudiado fue entre 18 y 19 años (47\%); 50,5\% presentaban cambios frecuentes de parejas; $48 \%$ mantenían relaciones sexuales desprotegidas; la percepción de riesgos de adquirir una ITS, tanto en el grupo de estudio y control fue de $23,2 \%$ y $40,3 \%$ respectivamente. ${ }^{4}$

En otro estudio realizado a 407 mujeres que consultaron en una clínica brasileña de planificación familiar para iniciar el uso de anticonceptivos mostró que el $35 \%$ tenían infecciones del aparato reproductivo. La vaginosis bacteriana VB (26\%) y clamidia $(7 \%)$ fueron las más comunes. ${ }^{5}$

En una investigación en el Perú sobre factores asociados al no uso de condón, estudio en adolescentes y adultos jóvenes de Chiclayo, los resultados fueron que la conducta sexual presenta diferencia en cuanto a la primera relación coital, siendo más precoces los varones. El uso adecuado del condón en los últimos seis meses fue mínimo en ambos sexos; la proporción de mujeres que refirieron que nunca lo habían usado fue muy alta. Se concluyó, con el análisis multivariado, que los factores asociados al no uso de condón en varones fueron la falta de disponibilidad, disminución del placer y relación amorosa. En las mujeres, pérdida de romanticismo, interferencia con la relación sexual y uso de otro método anticonceptivo. ${ }^{6}$

En la actualidad, el control de las ITS es difícil ya que al parecer intervienen otros factores, como los biológicos, ecológicos, además de los 
mencionados. Se subraya, que la frecuencia de las ITS ha aumentado considerablemente en los últimos años, con manifestaciones clínicas mínimas 0 atípicas.

Los diagnósticos clínicos y de laboratorio y el tratamiento correcto son necesarios; se debe tener presente que éste último no debe limitarse únicamente al paciente que consulta, sino también a los compañeros sexuales, si se pretende realizar un control máximo de estos pacientes y una profilaxis completa, tanto individual como colectiva. ${ }^{7}$

Esta situación de salud amerita el estudio científico de las conductas de riesgos y las infecciones de transmisión sexual en mujeres en edad fértil que acudieron al Centro de Salud La Esperanza en el distrito Alto de Alianza y al Centro de Salud San Francisco en el distrito Coronel Gregorio Albarracín Lanchipa de Tacna, en el año 2016.

\section{Material y métodos}

Por el tipo de aplicación, la investigación es básica, se utilizó un diseño no experimental, descriptivo y correlacional.

La población en estudio es finita y está conformada por las 3290 mujeres registradas en edad fértil en el Centro de Salud La Esperanza y 13963 en el Centro de Salud San Francisco que se atendieron en el año 2016. El tamaño muestral fue de 177 mujeres y para determinar el tamaño de la muestra se aplicó el método de muestreo al azar simple, usando la fórmula de muestra para poblaciones finitas.

Para la recolección de datos, en la variable conductas de riesgo, la técnica que se utilizó fue la encuesta, aplicando como instrumento un cuestionario en escala de Likert. Para medir la variable de infección de transmisión sexual, se usó la técnica documental aplicando como instrumento una ficha documental de resultados titulada Instrumento para medir las infecciones de transmisión sexual desarrollada por el Ministerio de Salud (MINSA) del Perú.

\section{Resultados}

De las 177 mujeres participantes de este estudio, se encontró que tienen ITS el 48,59 $\%$ del total de pacientes evaluadas, el 64,29\% corresponden al Centro de Salud San Francisco y el $34,41 \%$ al Centro de Salud La Esperanza (Tabla 1).
Tabla 1

Distribución por procedencia en mujeres en edad fértil

\begin{tabular}{|c|c|c|c|c|c|c|}
\hline \multirow{3}{*}{ ITS } & \multicolumn{4}{|c|}{ Procedencia } & \multirow{2}{*}{\multicolumn{2}{|c|}{ Total }} \\
\hline & \multicolumn{2}{|c|}{ San Francisco } & \multicolumn{2}{|c|}{ La Esperanza } & & \\
\hline & N. ${ }^{\circ}$ & $\%$ & N. ${ }^{\circ}$ & $\%$ & N. ${ }^{\circ}$ & $\%$ \\
\hline Sí & 54 & 64,29 & 32 & 34,41 & 86 & 48,59 \\
\hline No & 30 & 35,71 & 61 & 65,59 & 91 & 51,41 \\
\hline Total & 84 & 100 & 93 & 100 & 177 & 100 \\
\hline
\end{tabular}

Chi cuadrado: 15,771 Valor $p<0,001$

En el examen de secreción vaginal, en general el 24,29\% presenta un $\mathrm{KOH}$ positivo; el $6,78 \%$ presenta positivo a Trichomonas; el 14,69 $\%$, positivo a Pseudohifas (hongos); el 19,77\%, vaginosis bacteriana; el 27,68 \%, Gram Vaginal positivo; y un 12,99 \% presenta leucocitos más de 50 por campo (Tabla 2 ).

\section{Tabla 2}

Distribución por resultados de prueba de secreción vaginal en mujeres en edad fértil

\begin{tabular}{|c|c|c|c|}
\hline $\begin{array}{l}\text { Examen de } \\
\text { secreción } \\
\text { vaginal }\end{array}$ & & $\mathrm{N} .^{\circ}$ & $\%$ \\
\hline \multirow{2}{*}{$\mathrm{KOH}$} & Negativo & 134 & 75,71 \\
\hline & Positivo & 43 & 24,29 \\
\hline \multirow{2}{*}{ Trichomonas } & Negativo & 165 & 93,22 \\
\hline & Positivo & 12 & 6,78 \\
\hline \multirow{2}{*}{$\begin{array}{l}\text { Pseudohifas } \\
\text { (hongos) }\end{array}$} & Negativo & 151 & 85,31 \\
\hline & Positivo & 26 & 14,69 \\
\hline \multirow{2}{*}{$\begin{array}{l}\text { Células } \\
\text { clave }\end{array}$} & $\begin{array}{c}\text { Menos } 20 \% \text { células } \\
\text { clave }\end{array}$ & 142 & 80,23 \\
\hline & Más $20 \%$ células clave & 35 & 19,77 \\
\hline \multirow{2}{*}{$\begin{array}{l}\text { Gram } \\
\text { Vaginal } \\
\text { (Vaginosis } \\
\text { Bacteriana) }\end{array}$} & Negativo (0 a 6) & 128 & 72,32 \\
\hline & Positivo (7 a 10) & 49 & 27,68 \\
\hline \multirow{2}{*}{ Leucocitos } & $\begin{array}{c}\text { Negativo (menos de } 50 \\
\text { por campo) }\end{array}$ & 154 & 87,01 \\
\hline & $\begin{array}{l}\text { Positivo (más } 50 \text { por } \\
\text { campo) }\end{array}$ & 23 & 12,99 \\
\hline
\end{tabular}

En la distribución por procedencia, según factores sociodemográficos, del total de pacientes evaluados del Centro de Salud San Francisco, el $82,14 \%$ presenta 27 años a más, mientras que del total de pacientes evaluadas en el Centro de Salud La Esperanza, el 62,37 \%. Realizando la prueba estadística Chi cuadrado podemos encontrar un 
valor $p<0,05$. En cuanto al estado civil del total de pacientes del Centro de Salud San Francisco, el $52,38 \%$ es conviviente, mientras que del total de pacientes del Centro de Salud La Esperanza, el 58,06 \%. Realizando la prueba estadística Chi cuadrado podemos encontrar un valor $>0,05$. En cuanto a ocupación del total de pacientes del Centro de Salud San Francisco el 45,24 \% es comerciante, mientras que del total de pacientes del Centro de Salud La Esperanza, el 51,61\%. Realizando la prueba estadística Chi cuadrado podemos encontrar un valor $p<0,05$. En cuanto a número de hijos del total de pacientes del Centro de Salud San Francisco el 36,90 \% tiene 2 hijos, mientras que del total de pacientes del Centro de Salud La Esperanza, el 41,94 \%. Realizando la prueba estadística Chi cuadrado podemos encontrar un valor $p=0,05$.
En la distribución por procedencia, según conductas de riesgo, el número de parejas sexuales difiere entre el Centro de Salud San Francisco y el Centro de Salud La Esperanza con un valor $p<0,05$. En cuanto a con cuantas personas ha tenido relaciones sexuales, esta difiere entre el Centro de Salud San Francisco y el Centro de Salud La Esperanza con un valor $p<0,05$. En cuanto a relaciones sexuales con la persona que conoció por primera vez, difiere entre el Centro de Salud San Francisco y el Centro de Salud La Esperanza con un valor $p<0,05$. En cuanto a relaciones sexuales con una pareja que ha tenido varias parejas sexuales, difiere entre el Centro de Salud San Francisco y el Centro de Salud La Esperanza con un valor $p<0,05$. En cuanto a pareja estable o eventual, difiere entre el Centro de Salud San Francisco y el Centro de Salud La Esperanza con un valor $p<0,05$. En cuanto a predilección sexual hombre, mujer o ambos, no

\section{Tabla 3}

Distribución por factores sociodemográficos en mujeres en edad fértil

\begin{tabular}{|c|c|c|c|c|c|c|}
\hline \multirow{2}{*}{\multicolumn{2}{|c|}{ Factores sociodemográficos }} & \multicolumn{4}{|c|}{ Procedencia } & \multirow{3}{*}{$\mathrm{p}$} \\
\hline & & \multicolumn{2}{|c|}{ San Francisco } & \multicolumn{2}{|c|}{ La Esperanza } & \\
\hline & & $\mathrm{N} .^{\circ}$ & $\%$ & $\mathrm{~N} .^{\circ}$ & $\%$ & \\
\hline \multirow[t]{4}{*}{ Grupo de edad } & 15 a 18 años & 3 & 3,57 & 4 & 4,30 & \multirow{4}{*}{0,026} \\
\hline & 19 a 22 años & 4 & 4,76 & 13 & 13,98 & \\
\hline & 23 a 26 años & 8 & 9,52 & 18 & 19,35 & \\
\hline & 27 años a más & 69 & 82,14 & 58 & 62,37 & \\
\hline \multirow[t]{5}{*}{ Estado civil } & Conviviente & 44 & 52,38 & 54 & 58,06 & \multirow{5}{*}{0,068} \\
\hline & Casada & 9 & 10,71 & 5 & 5,38 & \\
\hline & Soltera & 18 & 21,43 & 9 & 9,68 & \\
\hline & Viuda & 1 & 1,19 & 1 & 1,08 & \\
\hline & Separada & 12 & 14,29 & 24 & 25,81 & \\
\hline \multirow[t]{4}{*}{ Ocupación } & Ama de casa & 34 & 40,48 & 19 & 20,43 & \multirow{4}{*}{0,017} \\
\hline & Comerciantes & 38 & 45,24 & 48 & 51,61 & \\
\hline & Empleadas & 9 & 10,71 & 18 & 19,35 & \\
\hline & Profesionales & 3 & 3,57 & 8 & 8,60 & \\
\hline \multirow[t]{6}{*}{$\mathrm{N}^{\circ}$ de hijos } & 0 hijos & 4 & 4,76 & 3 & 3,23 & \multirow{6}{*}{0,050} \\
\hline & 1 hijo & 24 & 28,57 & 36 & 38,71 & \\
\hline & 2 hijos & 31 & 36,90 & 39 & 41,94 & \\
\hline & 3 hijos & 19 & 22,62 & 15 & 16,13 & \\
\hline & 4 hijos & 6 & 7,14 & 0 & 0 & \\
\hline & 5 o más & 0 & 0 & 0 & 0 & \\
\hline
\end{tabular}


difiere entre el Centro de Salud San Francisco y el Centro de Salud La Esperanza con un valor $p>0,05$. En cuanto a predilección sexual o tipo de sexo, no difiere entre el Centro de Salud San Francisco y el Centro de Salud La Esperanza con un valor $p>0,05$. En cuanto a uso de preservativo, no difiere entre el Centro de Salud San Francisco y el Centro de Salud La Esperanza con un valor $p>0,05$. En cuanto a pensamiento que el hombre debe llevar la iniciativa, difiere entre el Centro de Salud San Francisco y el Centro de Salud La Esperanza, con un valor $p<0,05$.
Salud La Esperanza con un valor $p>0,05$. En cuanto a negación del uso del condón no difiere entre el Centro de Salud San Francisco y el Centro de Salud La Esperanza con un valor $p>0,05$. En cuanto a pareja con ITS no difiere entre el Centro de Salud San Francisco y el Centro de Salud La Esperanza con un valor $p>0,05$. En cuanto a flujo vaginal luego de relaciones sexuales no difiere entre el Centro de Salud San Francisco y el Centro de Salud La Esperanza con un valor $p>0,05$. En cuanto a acudir al médico luego de presentar flujo vaginal difiere entre el Centro de Salud San Francisco y el Centro

\section{Tabla 4}

Distribución por conductas de riesgo en mujeres en edad fértil

\begin{tabular}{|c|c|c|c|c|c|c|}
\hline \multirow[t]{3}{*}{ Conductas de riesgo } & & \multicolumn{4}{|c|}{ Procedencia } & \multirow{3}{*}{ Valor $p$} \\
\hline & & \multicolumn{2}{|c|}{ San Francisco } & \multicolumn{2}{|c|}{ La Esperanza } & \\
\hline & & $\mathrm{N} .^{\circ}$ & $\%$ & $\mathrm{~N} .^{\circ}$ & $\%$ & \\
\hline \multirow[t]{5}{*}{$\mathrm{N} .^{\circ}$ de parejas sexuales } & 1 pareja & 30 & 35,71 & 12 & 12,90 & \multirow{5}{*}{$<0,001$} \\
\hline & 2 parejas & 20 & 23,81 & 16 & 17,20 & \\
\hline & 3 parejas & 17 & 20,24 & 18 & 19,35 & \\
\hline & 4 parejas & 12 & 14,29 & 25 & 26,88 & \\
\hline & 5 parejas & 5 & 5,95 & 22 & 23,66 & \\
\hline \multirow{3}{*}{$\begin{array}{l}\mathrm{N} .^{\circ} \text { de parejas sexuales } \\
\text { durante los últimos } 3 \text { meses }\end{array}$} & 1 persona & 74 & 88,10 & 56 & 60,22 & \multirow{3}{*}{$<0,001$} \\
\hline & 2 personas & 7 & 8,33 & 22 & 23,66 & \\
\hline & $\begin{array}{l}3 \text { o más } \\
\text { personas }\end{array}$ & 3 & 3,57 & 15 & 16,13 & \\
\hline \multirow{2}{*}{$\begin{array}{l}\text { Relaciones sexuales con } \\
\text { persona que conoció por } \\
\text { primera vez }\end{array}$} & No & 77 & 91,67 & 67 & 72,04 & \multirow[b]{2}{*}{0,001} \\
\hline & Sí & 7 & 8,33 & 26 & 27,96 & \\
\hline \multirow{2}{*}{$\begin{array}{l}\text { Parejas sexuales con } \\
\text { comportamiento de riesgo o } \\
\text { promiscuas }\end{array}$} & No & 73 & 86,90 & 65 & 69,89 & \multirow{2}{*}{0,006} \\
\hline & Sí & 11 & 13,10 & 28 & 30,11 & \\
\hline \multirow[t]{2}{*}{ En la actualidad tiene pareja } & Eventual & 23 & 27,38 & 58 & 62,37 & \multirow{2}{*}{$<0,001$} \\
\hline & Estable & 61 & 72,62 & 35 & 37,63 & \\
\hline \multirow{3}{*}{$\begin{array}{l}\text { Uso de preservativo } \\
\text { Iniciativa del hombre }\end{array}$} & No & 18 & 21,43 & 0 & 0 & \multirow{3}{*}{$<0,001$} \\
\hline & Sí & 66 & 78,57 & 80 & 86,02 & \\
\hline & No precisa & 0 & 0 & 13 & 13,98 & \\
\hline
\end{tabular}

En la distribución por procedencia según factores de riesgo donde la iniciativa del uso del condón por parte del hombre no difiere entre el Centro de Salud San Francisco y el Centro de Salud La Esperanza, con un valor $p>0,05$. En cuanto a la mujer lleva preservativos, esta no difiere entre el Centro de Salud San Francisco y el Centro de Salud La Esperanza con un valor $p>0,05$. En cuanto a rechazo por no usar preservativos, no difiere entre el Centro de Salud San Francisco y el Centro de de Salud La Esperanza con un valor $p<0,05$. En cuanto a comunicación con su pareja acerca del flujo vaginal no difiere entre el Centro de Salud San Francisco y el Centro de Salud La Esperanza con un valor $p>0,05$. 
Tabla 5

Distribución por conductas de riesgo en mujeres en edad fértil

\begin{tabular}{|c|c|c|c|c|c|c|}
\hline \multirow{3}{*}{$\begin{array}{l}\text { Conductas de riesgo } \\
\text { Sintomatología de ITS } \\
\text { Tratamiento de ITS }\end{array}$} & & \multicolumn{4}{|c|}{ Procedencia } & \multirow{3}{*}{ Valor $p$} \\
\hline & & \multicolumn{2}{|c|}{ San Francisco } & \multicolumn{2}{|c|}{ La Esperanza } & \\
\hline & & $\mathrm{N} .^{\circ}$ & $\%$ & $\mathrm{~N} .^{\circ}$ & $\%$ & \\
\hline \multirow{3}{*}{$\begin{array}{l}\text { ¿Después de presentar flujo vaginal } \\
\text { mal oliente ardor o picazón en sus } \\
\text { partes genitales acudió a consulta } \\
\text { médica al centro o puesto de salud? }\end{array}$} & No & 61 & 75 & 44 & 92,47 & \multirow{3}{*}{0,001} \\
\hline & Sí & 23 & 25 & 49 & 7,53 & \\
\hline & No precisa & 0 & 0 & 0 & 0 & \\
\hline \multirow{3}{*}{$\begin{array}{l}\text { ¿Has tenido alguna infección de } \\
\text { transmisión sexual? }\end{array}$} & No & 61 & 72,62 & 44 & 47,31 & \multirow{3}{*}{0,001} \\
\hline & Sí & 23 & 27,38 & 49 & 52,69 & \\
\hline & No precisa & 0 & 0 & 0 & 0 & \\
\hline \multirow{3}{*}{$\begin{array}{l}\text { ¿Cumplió el tratamiento médico para } \\
\text { la ITS? }\end{array}$} & No & 64 & 76,19 & 56 & 60,22 & \multirow{3}{*}{0,023} \\
\hline & Sí & 20 & 23,81 & 37 & 39,78 & \\
\hline & No precisa & 0 & 0 & 0 & 0 & \\
\hline \multirow{3}{*}{$\begin{array}{l}\text { ¿Tu pareja(s) siguió el tratamiento } \\
\text { médico de la ITS? }\end{array}$} & No & 72 & 85,71 & 68 & 73,12 & \multirow{3}{*}{0,040} \\
\hline & Sí & 12 & 14,29 & 25 & 26,88 & \\
\hline & No precisa & 0 & 0 & 0 & 0 & \\
\hline \multirow{3}{*}{ Edad de inicio de relaciones sexuales } & 14 años & 10 & 11,90 & 6 & 6,45 & \multirow{3}{*}{0,000} \\
\hline & 15-17 años & 48 & 57,14 & 18 & 19,35 & \\
\hline & $18-20$ años & 26 & 30,95 & 69 & 74,19 & \\
\hline
\end{tabular}

En la distribución por ITS, según factores de riesgo, el número de parejas sexuales se asocia a ITS con un valor $p<0,05$. En cuanto a con cuantas personas ha tenido relaciones sexuales, se asocia a ITS con un valor $p<0,05$. En cuanto a relaciones sexuales con pareja que ha tenido varias parejas sexuales, se asocia a ITS con un valor $p<0,05$. En cuanto a pareja estable o eventual, no se asocia a ITS con un valor $p>0,05$. En cuanto al pensamiento que el hombre debe llevar condón, se asocia a ITS con un valor $p<0,05$. 


\section{Tabla 6}

Distribución por conductas de riesgo en mujeres en edad fértil

\begin{tabular}{|c|c|c|c|c|c|c|}
\hline \multirow{3}{*}{ Conductas de riesgo } & & \multicolumn{4}{|c|}{ ITS } & \multirow{3}{*}{$\begin{array}{c}\text { Valor } \\
\text { p }\end{array}$} \\
\hline & & \multicolumn{2}{|c|}{ Sí } & \multicolumn{2}{|r|}{ No } & \\
\hline & & $\mathrm{N} .^{\circ}$ & $\%$ & $\mathrm{~N} .^{\circ}$ & $\%$ & \\
\hline \multirow{5}{*}{$\begin{array}{l}\mathrm{N} .^{\circ} \text { de parejas } \\
\text { sexuales }\end{array}$} & 1 pareja & 11 & 26,19 & 31 & 73,81 & \multirow{5}{*}{0,044} \\
\hline & 2 parejas & 17 & 47,22 & 19 & 52,78 & \\
\hline & 3 parejas & 20 & 57,14 & 15 & 42,86 & \\
\hline & 4 parejas & 20 & 54,05 & 17 & 45,95 & \\
\hline & 5 parejas & 18 & 66,67 & 9 & 33,33 & \\
\hline \multirow{3}{*}{$\begin{array}{l}\mathrm{N} .^{\circ} \text { de parejas } \\
\text { sexuales en los } 3 \\
\text { últimos meses }\end{array}$} & 1 persona & 56 & 43,08 & 74 & 56,92 & \multirow{3}{*}{0.006} \\
\hline & 2 personas & 15 & 51,72 & 14 & 48,28 & \\
\hline & 3 o más personas & 15 & 83,33 & 3 & 16,67 & \\
\hline \multirow{2}{*}{$\begin{array}{l}\text { Parejas sexuales } \\
\text { con comportamiento } \\
\text { de riesgo o } \\
\text { promiscua }\end{array}$} & No & 68 & 46,15 & 21 & 53,85 & \multirow{2}{*}{0,031} \\
\hline & Sí & 18 & 49,28 & 70 & 50,72 & \\
\hline \multirow{3}{*}{$\begin{array}{l}\text { Uso de preservativo } \\
\text { por iniciativa del } \\
\text { hombre }\end{array}$} & No & 67 & 45,89 & 79 & 54,11 & \multirow{3}{*}{0,018} \\
\hline & Sí & 13 & 72,22 & 5 & 27,78 & \\
\hline & No precisa & 6 & 46,15 & 7 & 53,85 & \\
\hline
\end{tabular}

\section{Discusión}

Las infecciones de transmisión sexual tienen múltiples complicaciones en la salud sexual y reproductiva entre ellas infertilidad, transmisión materno infantil e infección por el virus del papiloma humano (VPH) que lleva a la consulta asistencial de un establecimiento a la población infectada, el diagnóstico precoz y tratamiento eficaz y oportuno resulta de gran valor teniendo en cuenta sus complicaciones y secuelas. La infección por clamidias trachomatis y la gonorrea son responsables de cervicitis en la mujer uretritis y enfermedad inflamatoria pélvica.

La población más sensible a contraer infecciones son los jóvenes y adolescentes. En el Perú, el inicio de relaciones sexuales cada vez a más corta edad constituye un factor de riesgo que repercute negativamente en los jóvenes y en la población por su alto índice de contagios a nivel nacional y mundial

La OMS calcula que, en la Región de las Américas, 51 millones de adolescentes y adultos (de 15 a 49 años) sexualmente activos contrajeron una ITS fácilmente curable en el 2012. La población más vulnerable a contraer infecciones son los jóvenes y adolescentes.

En el Perú el inicio de relaciones sexuales cada vez a más temprana edad constituye un factor de riesgo que repercute negativamente en los adolescentes. En este estudio se encontró que la mitad de las mujeres evaluadas presentaron alguna ITS $(48,59 \%)$, siendo mayor su proporción en el Centro de Salud San Francisco $(64,29 \%)$ y menor en el Centro de Salud La Esperanza $(34,41 \%)$.

En la distribución por ITS según factores de riesgo: piensas que la mujer debe esperar a que el hombre proponga la utilización del condón, piensas que si una mujer lleva preservativos en el bolso es una persona promiscua, que siempre está buscando sexo, alguna vez te ha rechazado tu pareja sexual por querer utilizar preservativos, cuándo tu pareja se ha negado rotundamente a utilizar condón, alguna vez has tenido relaciones sexuales con alguna pareja que tenía una infección de transmisión sexual, luego de tener relaciones sexuales alguna vez sintió flujo vaginal mal oliente, ardor o picazón en sus partes genitales, después de presentar flujo vaginal mal oliente, ardor o picazón 
en sus partes genitales acudió a consulta médica o centro o puesto de salud, habló con su pareja(s) del flujo vaginal mal oliente ardor o picazón de sus partes genitales, donde el ningún factor se asocia a ITS con un valor $p>0,05$.

En la distribución por ITS según factores de riesgo donde: ha tenido alguna infección transmisión sexual, cumplió el tratamiento médico para la ITS, la pareja(s) siguió el tratamiento médico de la ITS, alguna vez le hicieron un análisis de $\mathrm{VIH}$, alguna vez has sentido miedo ante la posibilidad de haberte infectado con el VIH, piensa que ha tenido riesgo de infectarte por VIH si no utilizas preservativo, alguna vez has tomado tanto alcohol que no recuerdas si utilizaste preservativo, tiene relaciones sexuales bajo la influencia de alcohol, hasta qué punto necesita tener relaciones sexuales, tiene relaciones sexuales bajo la influencia de drogas. Ningún factor se asocia a ITS con un valor $p<0,05$.

\section{Conclusión}

Las conductas de riesgo para las infecciones de transmisión sexual en mujeres en edad fértil corresponden a, número de parejas sexuales, relaciones sexuales con pareja que ha tenido varias parejas sexuales y pensamiento que el hombre debe llevar condón se asocia a ITS con un valor $p<0,05$.

La frecuencia de las infecciones de transmisión sexual en las mujeres en edad fértil que presentan síndrome de flujo vaginal corresponde a $48,59 \%$, donde el 64,29 \% corresponde al Centro de Salud San Francisco y el 34,41 \% al Centro de Salud La Esperanza.

Las conductas de riesgo en las mujeres en edad fértil corresponden a promiscuidad $y$ pensamiento que el hombre debe protegerse.

\section{Referencias}

1. Heredia B, Velazco A, Cutié E, Álvarez AB. Enfermedades de trasmisión sexual y su relación con el embarazo. Rev Cuba Obstet y Ginecol [Internet]. 1998 [cited 2021 Apr 6];24(1):28-33. Available from: http://scielo.sld.cu/scielo.php?script=sci_arttext\&pi$d=S 0138-600 X 1998000100005 \&$ Ing=es\&nr$\mathrm{m}=$ iso\&tlng=es

2. Sarampión R/. Enfermedades de Notificación Obligatoria [Internet]. [cited 2021 Apr 4]. Available from: www.ins.gob.pe

3. Organización Mundial de la Salud. Cada día, más de 1 millón de personas contraen una infección de transmisión sexual curable [Internet]. COMUNICADO DE PRENSA. 2019 [cited 2021 Apr 4]. Available from: https://www.who.int/es/ news/item/06-06-2019-more-than-1-millionnew-curable-sexually-transmitted-infectionsevery-day

4. Vidal $E$, Hernàndez $B$. Conductas sexuales de riesgo asociadas a las infecciones de transmisión sexual en adolescentes de una comunidad [Internet]. 2017 [cited 2021 Apr 6]. p. undefined. Available from: http://scielo.sld.cu/scielo.php?script=sci arttext\&pi$d=S 1729-519 \times 2017000400014 \& \overline{I n g}=e s$.

5. Teles E, Hardy E, Oliveira UM, Christopher J, Faùndes $\mathrm{E}$. Perspectivas internacionales de planificacion familiar [Internet]. 1998 [cited 2021 Apr 6]. Available from: https://www.guttmacher. org/sites/default/files/pdfs/pubs/journals/24spa02898.pdf

6. Soto V. Factores asociados al no uso del condón: Estudio en adolescentes y adultos jóvenes de Chiclayo. An la Fac Med [Internet]. 2006 [cited 2021 Apr 7];67(2):152-9. Available from: http://www.scielo.org.pe/scielo.php?script=sci arttext\&pid=S1025-55832006000200008\&I$\mathrm{ng}=\mathrm{es} \& \mathrm{nrm}=\mathrm{iso} \& \mathrm{t} \mathrm{ng}=\mathrm{es}$

7. Valdivia Luis, Escalante E. Manejo Sindrómico de las Enfermedades de Transmisión [Internet]. Dermatología Peruana UMSM. 2000 [cited 2021 Apr 6]. p. 1. Available from: https://sisbib. unmsm.edu.pe/bvrevistas/dermatologia/v10_ sup1/manejo_sind.htm

\section{Correspondencia:}

juanchangllio@gmail.com
Fecha de recepción: 01/06/2021

Fecha de aceptación: 02/08/2021 